\title{
Evaluation of Nanoparticle Inks on Flexible and Stretchable Substrates for Biocompatible Application
}

\author{
MartinSchubert \\ Electronics Packaging Laboratory \\ Technische Universität Dresden \\ Dresden, Germany \\ martin_schubert@tu-dresden.de \\ Lars Rebohle \\ Institute of Ion Beam Physics and \\ Materials Research \\ Helmholtz-Zentrum Dresden-Rossendorf \\ Dresden, Germany \\ 1.rebohle@hzdr.de
}

\author{
Yakun Wang \\ Electronics Packaging Laboratory \\ Technische Universität Dresden \\ Dresden, Germany \\ gabiwang@avt.et.tu-dresden.de \\ Marco Fritsch \\ Fraunhofer Institute for Ceramic \\ Technologies and Systems IKTS \\ Dresden, Germany \\ marco.fritsch@ikts.fraunhofer.de \\ Karlheinz Bock \\ Electronics Packaging Laboratory \\ Technische Universität Dresden \\ Dresden, Germany \\ karlheinz.bock@tu-dresden.de
}

\author{
Mykola Vinnichenko \\ Fraunhofer Institute for Ceramic \\ Technologies and Systems IKTS \\ Dresden, Germany \\ mykola.vinnichenko@ikts.fraunhofer.de \\ Thomas Schumann \\ Institute of Ion Beam Physics and \\ Materials Research \\ Helmholtz-Zentrum Dresden-Rossendorf \\ Dresden, Germany \\ t.schumann@hzdr.de
}

\begin{abstract}
The flexible and stretchable electronic market is increasing particularly in the field of biomedical electronics. Widely used printed silver conductive tracks today are only eligible for on-skin applications. However, for biomedical applications fully biocompatible, flexible and even stretchable materials for device fabrication are needed. This paper presents an additive printing approach to fabricate flexible and stretchable electronics by using a biocompatible platinum material. Usually, in order to realize electrically conducting Ptinterconnects by inkjet printing, it requires a furnace sintering at prohibitively high temperatures, which are not compatible with thermal sensitive polymeric substrates. This paper describes a high-power diode laser sintering (HPDL) and a flash lamp annealing (FLA) as promising alternative sintering methods. Both processes are eligible whereas laser sintering showed slightly better results. Bending tests and adhesive strength tests of platinum printed inks on polyimide with up to 180000 cycles, show that printed platinum is a suitable biocompatible material for flexible electronics.
\end{abstract}

Keywords-flexible, stretchable, biocompatible, photonic sintering, platinum, silver, nanoparticle ink, flash lamp, laser sintering

\section{INTRODUCTION}

The current IDTechEx-Roadmap for stretchable and conformal electronics forecasts a commercial market of over 800 million USD by 2028 [1]. Particularly biomedical applications are of high interest considering todays aging society $[1,2]$. When focusing on telemedical topics such as long-term remote monitoring of patient's health parameters one can distinguish two main application demands. The first one is extracorporeal, non-invasive electronics such as smart skin and wearable electronics. The second one is intracorporeal and includes invasive applications e.g. pressure sensors for stent grafts [3] or intraoral sodium sensors [4]. However, considering

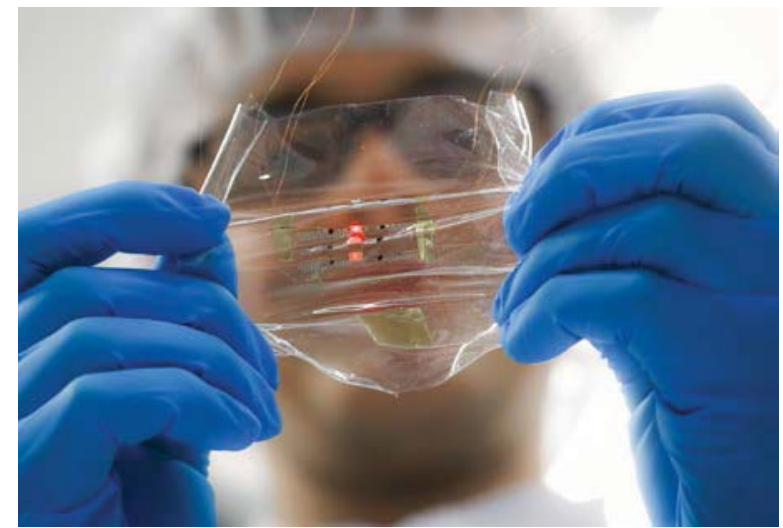

Fig. 1 Stretchable printed Ag-tracks on a flexible and stretchable substrate with examplaric assembled LED.

the choice of materials, the biocompatibility is, besides the mechanical stability, of vital importance in both cases. Most research articles report results for biocompatible polymers such as PDMS, polyurethane, polyethylene and others as substrate materials and conductive fillers (metal wires, carbon black, carbon nanotubes), silver nanoparticles or nanowires for conductive purposes [5-8]. Fig. 1 exemplarily shows a stretchable biocompatible substrate with a printed silver nanoparticle ink during stretching of ca. $20 \%$. However, silver is well known for its cytotoxic and antibacterial effect and therefore is limited to extracorporeal usage. This is also the case, if silver is embedded in polymeric encapsulations, since polymers are non-hermetic and permeable to ions $[9,18]$. Therefore, there is a need for electronics constituted of biocompatible materials only. Besides biocompatible polymers, noble metals with low electrical resistivity such as Au or Pt are often used $[10,11]$. In this context, additively manufactured platinum conductive tracks represent a promising approach to 
avoid the usage of cytotoxic silver materials. However, there are no known solutions for printed platinum layers for biocompatible flexible and stretchable applications available at the moment. The reason is that platinum has a relatively high melting temperature of $1768{ }^{\circ} \mathrm{C}$, which leads to high sintering temperature, exceeding the maximum allowed temperature of most stretchable polymeric substrates. Additive processing, such as inkjet printing, shows advantages with regard to individual manufacturing of medical devices without additional mask and chemical processing. This paper presents inkjet printed platinum interconnects and their application for biomedical electronics. A comparison of properties like conductivity and bending reliability between platinum and silver, as state of the art material, will be discussed. To enable the platinum ink processing on low temperature polymeric substrates the impact of fast photonic sintering methods on the interconnect properties will be presented.

\section{EVALUATION OF MATERIALS}

\section{A. Inkjetprinting of nanoparticle inks on reference ceramic substrate}

In this work water-based silver (Ag-LT-20) and platinum (Pt-LT-20) nanoparticle inks, both from Fraunhofer Institute for Ceramic Technologies and Systems (IKTS) were used. The specifications of the inks are shown in TABLE I. The inks were printed with an inkjet printer Pixdro LP50 from Meyer Burger using a Dimatix Cartridge (10 pl) printhead.

TABLE I. PROPERTIES OF NANOPARTICLE INKS

\begin{tabular}{|c|c|c|}
\hline & AG-LT-20 & PT-LT20 \\
\hline Nanoparticles size & $<200 \mathrm{~nm}$ & $<200 \mathrm{~nm}$ \\
\hline Solvent & water based & water based \\
\hline $\begin{array}{l}\text { Solid content wt.- } \\
\%\end{array}$ & 20 wt.- $\%$ & 20 wt.- $\%$ \\
\hline Surface tension & $34 \mathrm{mNm}$ & $38 \mathrm{mNm}$ \\
\hline $\begin{array}{l}\text { Viscosity } \\
100 / \text { sec, } 22{ }^{\circ} \mathrm{C}\end{array}$ & $8 \mathrm{mPas}$ & $12 \mathrm{mPas}$ \\
\hline
\end{tabular}

As a reference layout to determine the electrical resistance of the printed interconnects before and after sintering, a rectangular pattern of $15 \mathrm{~mm} \times 1 \mathrm{~mm}$ with varying number of layers at a print resolution of $600 \mathrm{dpi}$ is introduced. The interconnect thickness is $0.9 \mu \mathrm{m}$ for 3 layer $(3 \mathrm{~L})$ and $1.4 \mu \mathrm{m}$ for 5 layer (5L). Fig. 2 shows this test layout on $\mathrm{Al}_{2} \mathrm{O}_{3}$ substrates, allowing thermal sintering at peak temperatures of (100$500)^{\circ} \mathrm{C}$. The sintering parameters were set constant on a hotplate for a time of two hours. The resistance of the ink was measured with a Cascade Microtech PM8 probestation and Keysight B1500A semiconductor device analyzer along the printing direction, and is shown in Fig. 3. The results show higher resistance for the 3 layer structure compared to the 5 layer structure. As expected, with increasing sintering temperature, the resistance decreases with a leveling slope at $250{ }^{\circ} \mathrm{C}$ to about $93 \Omega(5 \mathrm{~L})$ and $156 \Omega(3 \mathrm{~L})$ which equals approx. 80 times bulk platinum resistance. This matches with the recommendations for sintering temperatures of the ink

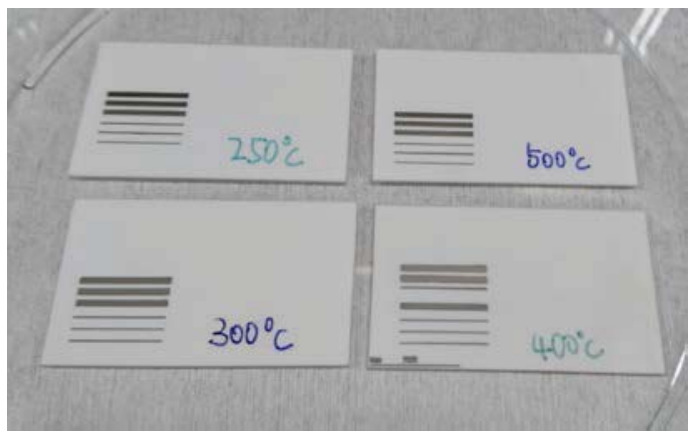

Fig. 2 Standard Pt-print-pattern on $\mathrm{Al}_{2} \mathrm{O}_{3}$ substrates thermally sintered at different sintering temperatures.

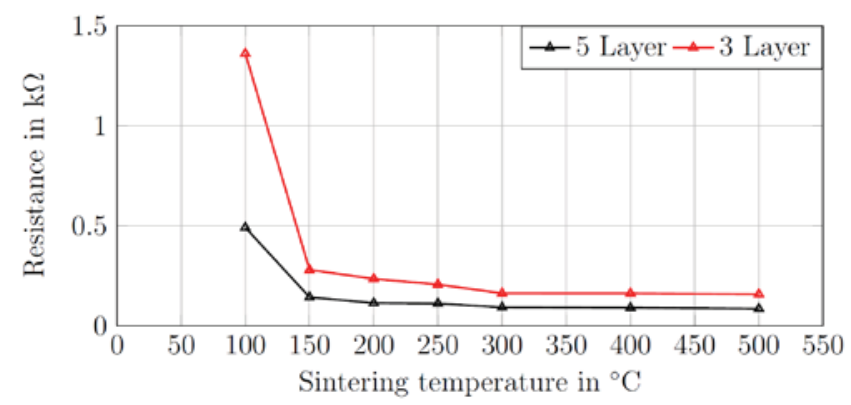

Fig. 3 Resistance of printed platinum interconncets on reference ceramic $\mathrm{Al}_{2} \mathrm{O}_{3}$ susbtrate.

manufacturer. Silver ink starts sintering at much lower temperatures $\left(50{ }^{\circ} \mathrm{C}\right)$ and reaches its optimum of resistance at around $150{ }^{\circ} \mathrm{C}$ with a resistance of ca. $1,6 \Omega(5 \mathrm{~L})$ and $1,8 \Omega$ $(3 \mathrm{~L})$, which is approx. 10 times bulk silver resistivity.

\section{B. Biocompatibility}

International standards like DIN EN ISO 10993 and USP Class VI describe distinct tests for the evaluation of the biocompatibility of materials. The ink materials used in this work are new research products and were neither classified by the manufacturer, nor tested according to such standards. Therefore, both nanoparticle inks were tested on in-vitro cytotoxicity according to DIN EN ISO 10993-5. For this test, vital human cells (cell line HFFF2) were grown on the printed samples of the ink. The ink was fully printed on glass slides and sintered at the temperatures $250^{\circ} \mathrm{C}(\mathrm{Pt})$ and $150{ }^{\circ} \mathrm{C}(\mathrm{Ag})$ for 2 hours as specified in the ink datasheet. Additionally, glass slides without ink serve as positive reference specimen with $100 \%$ cell vitality. The samples were incubated for 2 days together within a cell culture medium. After that procedure, resazurin (blue dye), is applied to the cells and incubated for another 5 hours. Vivid cells metabolize the blue dye into resorufin (pink dye). Depending on the number of living cells on the printed ink, the color of the dye varies and the vitality of the cells can be evaluated by using a photometer. Reduced cell vitalities by more than $30 \%$ compared to the positive reference is considered as cytotoxic effect of a material. The test was performed 3 times within 3 runs with 3 samples of each material per run. Fig. 5 shows the biomedical compatibility of printed platinum ink compared to silver ink. The results show, 


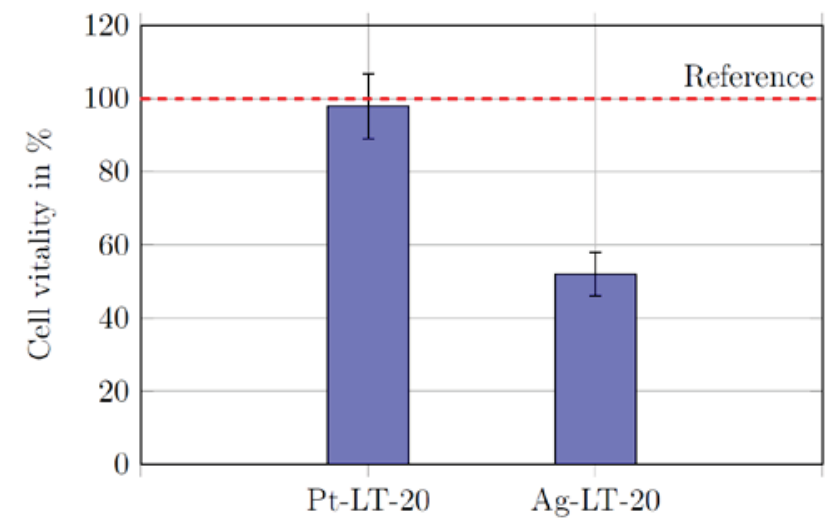

Fig. 4 Biocompatibility test of printed platinum and silver ink according to in-vitro cytotoxicity test DIN EN ISO 10993-5 with cell line HFFF2 on glass.

that such printed platinum nanoparticle inks and films are able to form highly biocompatible surfaces when sintered.

\section{Pt ink evaluation on polymer substrates}

Flexible and stretchable electronics are often fabricated on polymeric substrate materials. In this paper polyimid PI-2611 from HD Microresist was used for flexible application due to its promising material properties. The glass transition temperature of PI-2611 is of $360^{\circ} \mathrm{C}$, that is rather high value as for polymer material, and the coefficient of thermal expansion (CTE) is $3 \mathrm{ppm} /{ }^{\circ} \mathrm{C}$ [15]. Furthermore, this polyimide is typically used for biomedical applications and flexible circuit boards today $[9,12]$. In order to realize a foil of an average thickness of $9.5 \mu \mathrm{m}$, the polymer has been spin coated on a silicon wafer, cured and delaminated. For flexible applications the material combination of platinum ink and PI-2611 was tested after thermal sintering. For stretchable applications a poly(carbon urethane) ChronoFlex $\mathrm{AR}^{\circledR}$ from AdvanSource Biomaterials was chosen due to its more hydrophilic surface properties with respect to ink wetting, which makes its printing much easier compared to PDMS substrates. AdvanSource has classified Chronoflex $\mathrm{AR}^{\circledR}$ according to USP Class VI for short term implantation [13]. Chronoflex $\mathrm{AR}^{\circledR}$ has a CTE between (101-161) ppm $/{ }^{\circ} \mathrm{C}$ [16]. The manufacturer does not provide the maximum process temperature of the polymer. Therefore, it was tested by evaluating the softness and adhesion during heating in several steps. For the temperatures above $120^{\circ} \mathrm{C}$ the polymer sticks to the wafer and can not be removed from the wafer surface. Therefore, $120{ }^{\circ} \mathrm{C}$ was considered as the maximum process temperature for this polymer. In order to realize a foil with an average thickness of $12 \mu \mathrm{m}$ the material was spin coated on wafer. Alternatively, ChronoFlex $\mathrm{AR}^{\circledR}$ can be combined to a multilayer foil by adding a layer of Sylgard ${ }^{\circledR}$ 184 from Dow Corning. Due to the maximum temperature limitation this material requires other sintering methods then conventional thermal treatment in furnace, which will be considered in section IV.

Pt shows an approx. 100 times higher electrical resistance compared to $\mathrm{Ag}$ after thermal sintering at $250{ }^{\circ} \mathrm{C}$. The test on cytotoxicity, however, indicates that printed $\mathrm{Pt}$ is much more suitable for direct cell contact application than Ag. The chosen substrate materials are considered as suitable for biocompatible applications.

\section{RESUlts OF PLATINIUM ON FLEXIBLE SUBSTRATES}

\section{A. Bending test evaluation}

For bend testing a test setup was used which was already shown in previous publications $[12,14]$. The setup consists of a sample holder belt, which is connected on one end to a motor and on the other end to a weight (Fig. 5). The latter causes the required tension in the holder while moving. An exchangeable reel enables the bending with a preset radius. Simultaneously, change of interconnect resistance was measured by applying a certain constant current, depending on the structure resistance range of $(9.9,0.5$ or 0.18$) \mathrm{mA}$, to measure to the test structures, which are connected with $\mathrm{H} 20 \mathrm{E}$ conductive adhesive from EPOTEK and copper wires. By monitoring the voltage change using a data acquisition board (DAQ) the electrical resistance is logged. The test samples were printed interconnects on polyimide with $70 \mathrm{~mm}$ length and two pixel width. The platinum film width depends on the printed layer number and was corresponds to ca. $130 \mu \mathrm{m}$ on polyimide substrate. All samples were printed on polyimide and thermally sintered at $250{ }^{\circ} \mathrm{C}$ for $2 \mathrm{~h}$. The used bending radius was $5 \mathrm{~mm}$.

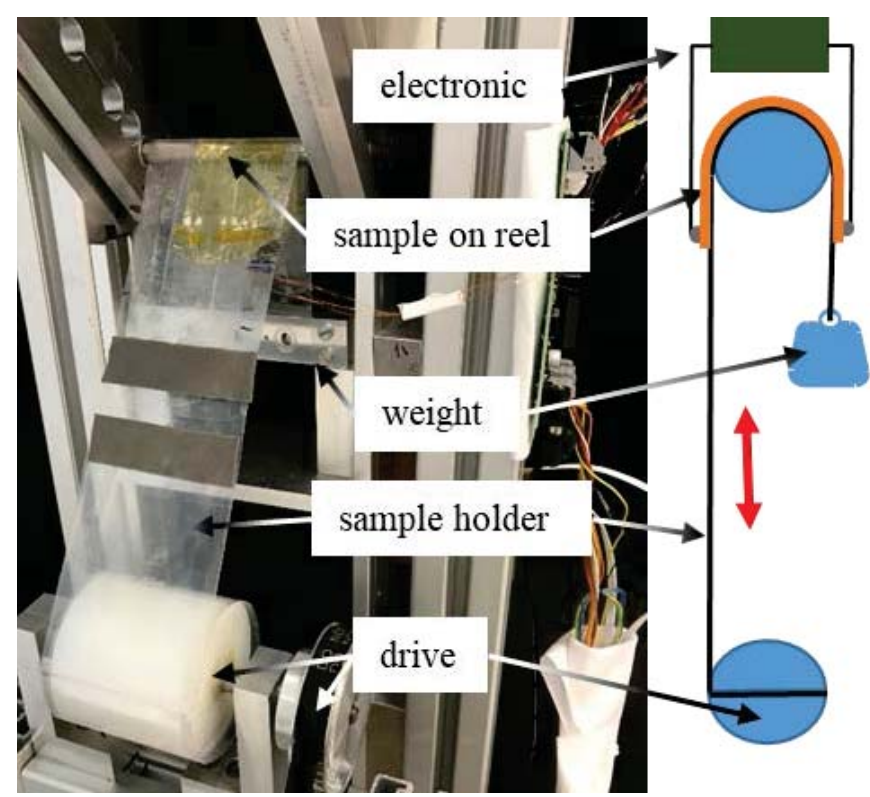

Fig. 5 Bending test rig for foil substrates with resitstance monitoring.

TABLE II. BENDING CYCLES AS AVERANGE OF 5 STRUCTURES OF 3 DIFFERENT LAYER

\begin{tabular}{|l|c|c|c|}
\hline Pt - line structures & 1 layer & 3 layer & 5 layer \\
\hline Resistance before test & $20,5 \mathrm{k} \Omega$ & $7,3 \mathrm{k} \Omega$ & $4,3 \mathrm{k} \Omega$ \\
\hline \multirow{3}{*}{$\begin{array}{l}\text { Increased resistance at } \\
\text { end of test }\end{array}$} & - & $5 \%$ & $7 \%$ \\
\cline { 2 - 4 } & $1 \%$ & $5 \%$ & $5 \%$ \\
\cline { 2 - 4 } & $1 \%$ & $4 \%$ & $5 \%$ \\
\cline { 2 - 4 } & $1 \%$ & $4 \%$ & $4 \%$ \\
\cline { 2 - 4 } & $1 \%$ & $4 \%$ & $3 \%$ \\
\hline $\begin{array}{l}\text { Max. number of cycles at } \\
\text { end of test }\end{array}$ & 204368 & 189582 & 182380 \\
\hline
\end{tabular}


TABLE II. shows the results of bending cycling test for Pt layers. All samples lasted during minimum 182000 cycles without total failure. However, a dependency of the cycling behavior on the sample thickness (number of layers) can be seen. For all samples the resistance increased during the test but did not exceed $7 \%$. Thicker samples show higher increase in resistance than thinner. However, the latter have a much higher resistance and therefore are only suitable for certain applications. Compared to bending test results in literature where 5 out of $7 \mathrm{Ag}$ printed tracks withstood more than 200000 bending cycles (radius $3 \mathrm{~mm}$ ) with a resistance increase of (6070) \% [19], the Pt test structures showed good characteristics.

\section{B. Adhesive strenght test evaluation}

The adhesive strength of thermally sintered platinum was evaluated according DIN EN 4624. The stud pull test was performed using a Condor Sigma bondtester from XYZTECH. The platinum ink was printed on polyimide substrate mounted on a wafer and thermally sintered at $250^{\circ} \mathrm{C}$ for $2 \mathrm{~h}$. An adhesive glue PD955M from Heraeus with a thickness of $200 \mu \mathrm{m}$ was applied using stencil printing. After curing at $100^{\circ} \mathrm{C}$ for 15 min the stud-pull testing setup (Fig. 6) adapted from [17] was visually placed over the printed adhesive pattern. The studs were previously fixed in correct height and perpendicular within the setup. After curing of the adhesive, the weight and the fence for studs were carefully removed and the setup was placed in the bondtester. The contact area of the stud was $38.5 \mathrm{~mm}^{2}$. After pulling, only adhesion failures at the interface between the substrate and the platinum ink occurred. The impact of $\mathrm{O}_{2}$ plasma treatment with regard to enhancing adhesion was characterized by applying $\mathrm{O}_{2}$ plasma for $2 \mathrm{~min}$ at $600 \mathrm{~W}$ in a DREVA CLEAN 450 Plasmaoven from VTD Vakuumtechnik Dresden GmbH.

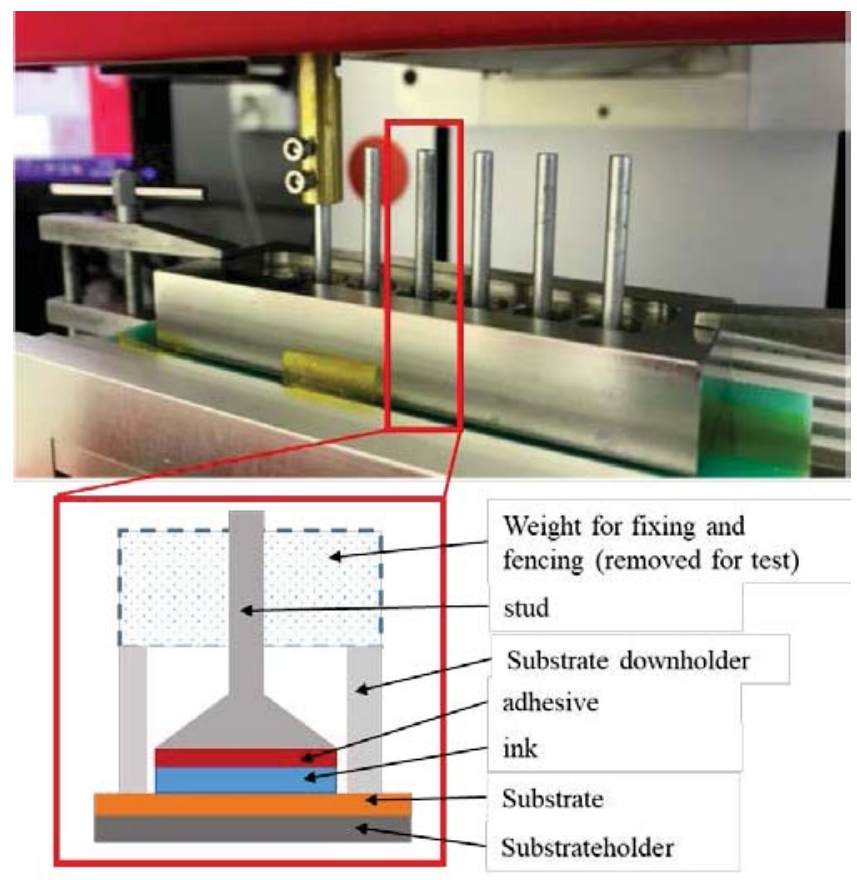

Fig. 6 Adhesive strength stud-pull testing setup.

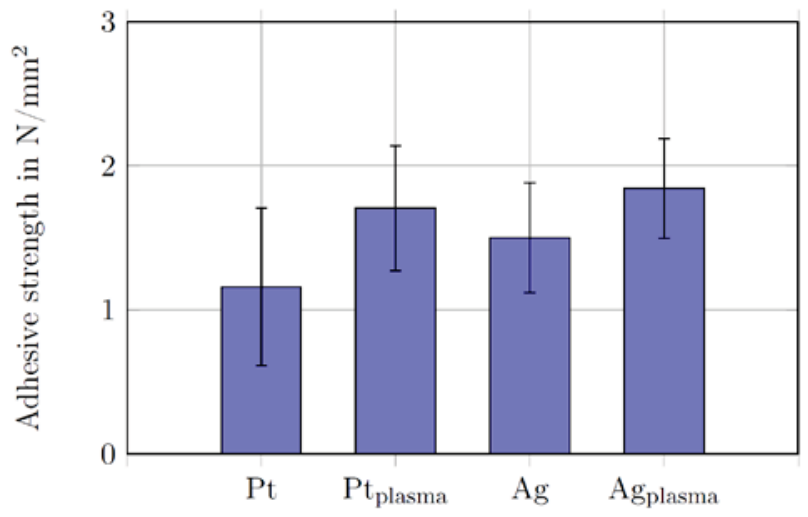

Fig. 7 Adhesive strength of printed Pt and Ag inks on PI-2611 with and without $\mathrm{O}_{2}$ plasma treatment for anhanced adhesion.

Fig. 7 shows results on the pull test of Pt and Ag ink on the polyimide substrate. Within the standard deviation Pt printed films showed a slightly lower adhesive strength in comparison to Ag. Plasma treatment enhances the adhesion for both inks as expected and Pt ink achieves nearly the same results as $\mathrm{Ag}$ films. In comparison to sputtered $\mathrm{Cu}$ on cyclic olefin copolymer (COC), the printed Pt films without plasma treatment showed $25-35 \%$ less adhesion strength [18]. However, it is expected that such adhesion can be further improved by ink development, by using appropriate organic binders.

\section{SINTERING ON LOW TEMPERATURE SUBSTRATES}

In this paper two sintering methods on the millisecond time-scale have been tested in order to enable electrically conductive Pt-interconnects. The energy of the incident light is selectively absorbed by the printed structures leading to their localized very rapid heating without damaging the thermally sensitive substrate. For this evaluation printed structures as described in section II have been used with flash lamp Chronoflex or high-power diode laser (HPDL) treatment. The resistance of the initial samples after drying at $50^{\circ} \mathrm{C}$ were generally higher than $1 \mathrm{M} \Omega$, ranging up to more than $20 \mathrm{M} \Omega$.

\section{A) Flash Lamp Annealing}

The flash lamp system as shown in Fig. 8 consists of a bank of xenon lamps and a reflector, which allows the application of short high energy pulses on a wafer-like substrate.

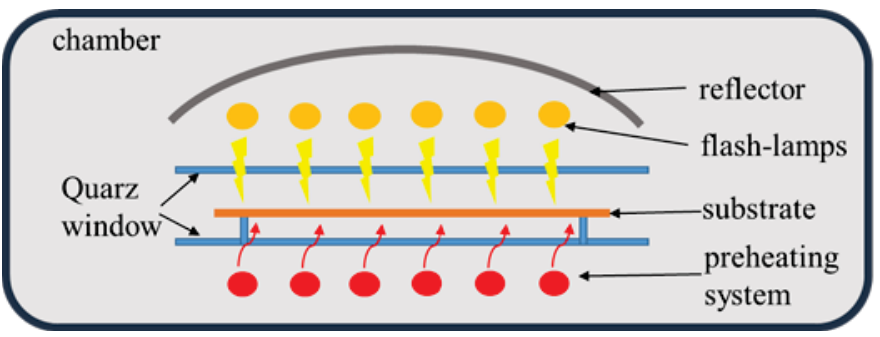

Fig. 8 Schematic drawing of the flash lamp unit (adapted from [20]). 


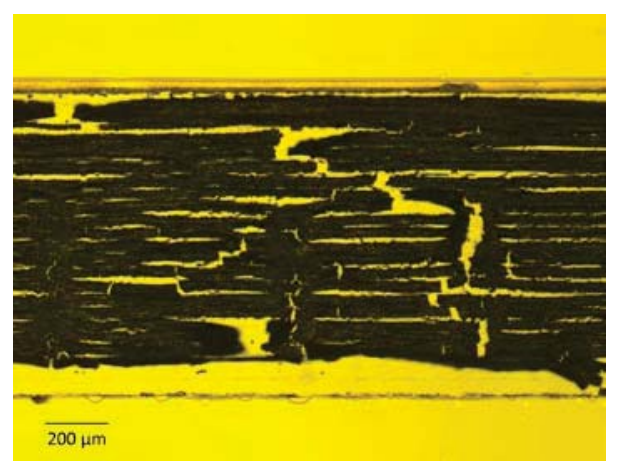

$5 \mathrm{~L}$ resistance: varying (1-3) $\mathrm{k} \Omega$

(a)

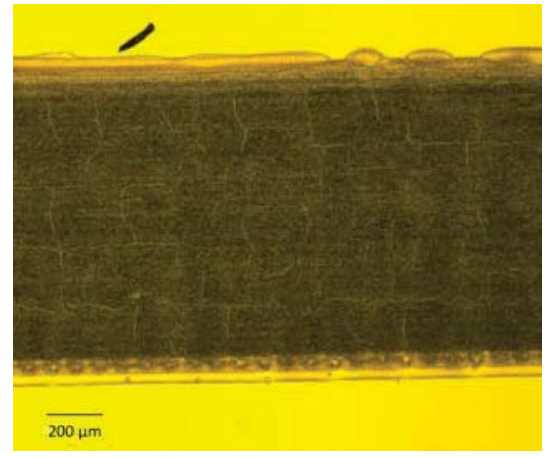

$5 \mathrm{~L}$ resistance: $350 \mathrm{k} \Omega$

(b)

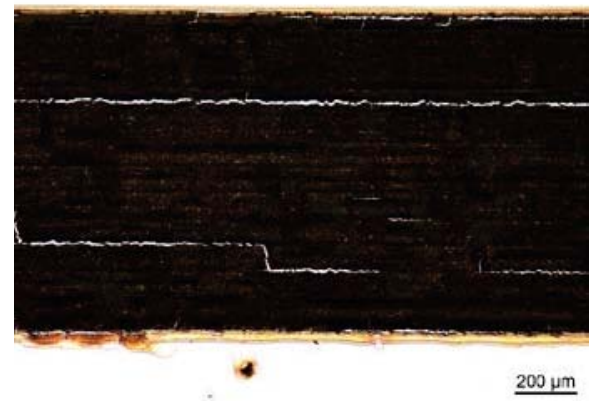

$5 \mathrm{~L}$ resistance: $180 \Omega$

(c)

Fig. 9 Microcopic images of FLA sintered (a,b) and HPDL line laser sintered (c) 5 layer Pt structures.

The processing was performed with energy densities of $(1.28-30) \mathrm{J} / \mathrm{cm}^{2}$ and flash times between $(0.5-20) \mathrm{ms}$ for single and multiple flashes after a recharging time of several minutes.

The best results were achieved with the parameters shown in TABLE III. The challenge was to find the right balance between energy and flash time. If the thermal budget is too low, no sintering effect appears, whereas a too high thermal budget causes the Pt ink to burn off. Two approaches have been considered. Fig. 9 (a) shows the first approach using a low energy and a short flash time but only one flash. This results in a cracked layer with generally high, inconstant resistance. The second approach used a longer flash time with a slightly higher energy density. The result in Fig. 9 (b) shows a Pt-layer with few cracks after 5 flashes. This indicates that more flashes at low energy might lead to improved layer quality and better properties.

TABLE III. PARAMETER SET FOR FLA SINTERING

\begin{tabular}{|l|c|c|}
\hline \multicolumn{1}{|c|}{ FLA } & $\begin{array}{c}\text { Chronoflex } \\
\text { Fig. 9 (a) }\end{array}$ & $\begin{array}{c}\text { Chronoflex } \\
\text { Fig. 9 (b) }\end{array}$ \\
\hline Energy density $\left(\mathrm{J} / \mathrm{cm}^{2}\right)$ & 1.28 & 1.8 \\
\hline Flash time $(\mathrm{ms})$ & 0.5 & $5 \times 1.3$ \\
\hline
\end{tabular}

\section{B) High power diode laser processing}

For laser sintering, a novel approach based on a micro-optically optimized one-dimensional diode laser source (diode laser array, LIMO $\mathrm{GmbH}$ ) having a line-shaped beam profile was used (Fig. 10). A near-uniform, non-Gaussian energy density distribution in the plane of the sample is realized in this way. The length of the diode laser array, and correspondingly the laser line length on the sample surface, was $30 \mathrm{~mm}$. Compared to the standard point laser, the diode laser array provides certain advantages because of its large scan area which prevents the discontinuous heat transfer and most importantly a inhomogeneity in the processed area. Semiconductor diode lasers with emission in the near infrared spectral range wavelength $980 \mathrm{~nm}$ ) were used. The processing was performed

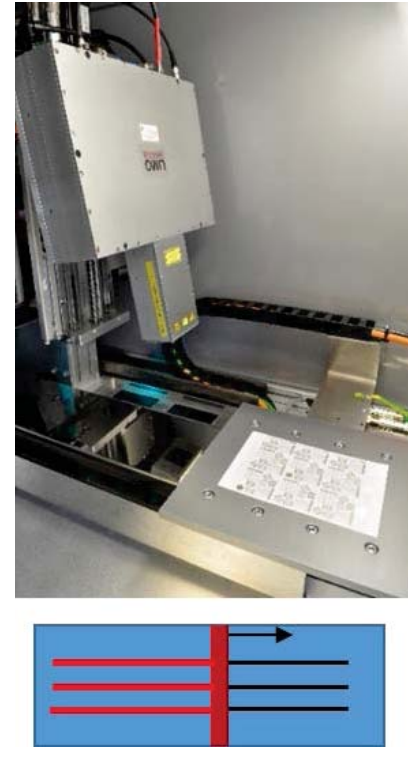

Fig. 10 Diode laser array facility and schematic representation of the processing.

TABLE IV. PARAMETER SET FOR LASER SINTERING

\begin{tabular}{|l|c|}
\hline Laser Parameter & $\begin{array}{c}\text { Chronoflex } \\
\text { Fig. 9 (c) }\end{array}$ \\
\hline Energy density $\left(\mathrm{J} / \mathrm{cm}^{2}\right)$ & 1.1 \\
\hline Process speed $\left(\mathrm{mm} / \mathrm{min}^{2}\right)$ & 10000 \\
\hline Dwell time $(\mathrm{ms})$ & 0.6 \\
\hline Power density $\left(\mathrm{W} / \mathrm{cm}^{2}\right)$ & 1867 \\
\hline 5 layer resistance $(\mathrm{Ohm})$ & 180 \\
\hline
\end{tabular}

using a continuous substrate movement (R2R compatible) with a speed in the range of $(6-25) \mathrm{m} / \mathrm{min}$ (dwell-time (0.2-1) $\mathrm{ms}$ ) at a laser power density of either $1.9 \mathrm{~kW} / \mathrm{cm}^{2}$ or $3.4 \mathrm{~kW} / \mathrm{cm}^{2}$.

The best results were achieved with parameters shown in TABLE IV. Using HPDL processing at energy densities within the range $(0.7-1.2) \mathrm{J} / \mathrm{cm}^{2}$ allows minimizing such issues with 
Pt-interconnects as delamination and cracks. In this case layers were showing promising electrical properties since only singular cracks appear along the printing direction. Although the minimum achieved electrical resistance is still factor two higher compared to that of thermally sintered layers, there is still a room for its further decreasing by optimizing laser parameters in order to completely avoid the occurrence of the cracks.

\section{CONCLUSION}

This paper contributes to a further development of a promising approach of biocompatible flexible and stretchable additively manufactured electronics using only biocompatible materials. In this respect, regarding biocompatible applications, $\mathrm{Pt}$ ink is a favorable material to use compared to state of the art $\mathrm{Ag}$ inks. The results after thermal sintering seem to be promising for flexible applications, even if the ohmic losses in case of Pt are higher than in case of Ag. The bending results of printed Pt films showed a superior film stability during more than 180.000 bending cycles on $5 \mathrm{~mm}$ radius, which is comparable to $\mathrm{Ag}$ ink results known in literature. The initial difference in lower adhesive strength of $24 \%$ between Pt and $\mathrm{Ag}$, was significantly reduced to $8 \%$ by using a plasma pretreatment of the substrate.

In this paper photonic curing such as FLA and HPDL was tested to overcome the problem of the Pt-ink sintering on flexible substrates. The used FLA configuration showed promising results, however, the systems energy parameters could not be sufficiently adapted to achieve fully intact interconnects. The present results show that the FLA setups capable of providing low energetic (around $1 \mathrm{~J} / \mathrm{cm}^{2}$ ) pulses with high frequency should be tested in order to provide results comparable to those of laser sintering. HPDL line laser processing showed a superior film sintering with lower film resistance in comparison to the tested FLA setup. However, both methods and the Pt ink have to be further optimized, to reduce crack building during heat treatment.

\section{ACKNOWLEDGMENT}

The authors would like to thank Antje Beyer and Prof. Günter Vollmer from the chair of Molecular Cell Physiology and Endocrinology of TU Dresden for providing the equipment for cytotoxicity tests. The authors would further like to thank the Group of Prof. Elfadel of the Masdar Institute, AbuDhabi in the frame of the Twinlab for fruitful discussions.

This work was supported by the European Social Fund (ESF) and the Free State of Saxony.

FhG IKTS acknowledges partial financial support by Fraunhofer-Attract Project INNOVELLE.

\section{REFERENCES}

[1] K. Ghaffarzadeh, J. Hayward,X. He, "Stretchable and Conformal Electronics 2018-2028 - Materials, components, products and 10-year market outlook", IDTechEx, viewed on 06.06.2018

[2] Z.Wu, Y. Huang, R. Chen, "Opportunities and Challenges in Flexible and Stretchable Electronics: A Panel Discussion at ISFSE2016, Micromachines, MDPI, vol. 8 (4):129, 2017

[3] S. Kirsten et al., "Biocompatible packaging for implantable miniaturized pressure sensor device used for stent grafts: Concept and choice of materials", IEEE 16th Electronics Packaging Technology Conference (EPTC), pp. 719 - 724, 2014

[4] Y. Lee et al.," Wireless, intraoral hybrid electronics for real-time quantification of sodium intake toward hypertension management", Proceedings of the National Academy of Sciences, vol. 115 (21), 2018

[5] M. Shi et al., "Self Powered Analogue Smart Skin", ACS Nano, vol. 10, pp. 4083-4091, 2016

[6] D.-H. Kim et al.: "Epidermal Electronics", Science, vol. 333, pp. 838843,2011

[7] J. Kim et al.: "Stretchable silicon nanoribbon electronics for skin prosthesis", Nature Communications vol. 5, Article number: 5747 (2014)

[8] M. Park, J. Park, U. Jeong," Design of conductive composite elastomers for stretchable electronics", Nano Today, vol. 9(2), 2014

[9] B. Rubehn, T. Stieglitz, "In vitro evaluation of the long-term stability of polyimide as a material for neural implants", Biomaterials, vol. 31, pp. 3449-3458, 2010.

[10] M. Schuettler et al., "Stretchable tracks for laser-machined neural electrode arrays", IEEE International Conference of the Engineering in Medicine and Biology Society, 2009

[11] Y. Wang et al., "Stretchable Platinum Network-Based Transparent Electrodes for Highly Sensitive Wearable Electronics, Small, vol. 13(27), 2017

[12] HD MicroSystems ${ }^{\mathrm{TM}}$, "PI-2600 Series - Low Stress Applications", Technical Information, http://www.dupont.com/content/dam/dupont/ products-and-services/electronic-and-electrical-materials/semiconductorfabrication-and-packaging-materials/documents/PI-2600_ProcessGuide .pdf, 2009, viewed on 12.06.2018

[13] M. Schubert, H. Berg, S. Friedrich, K. Bock, "Evaluation of Dispensed Carbon Nanotube Ink on Flexible Substrates for Biocompatible Application", 6th Electronic System-Integration Technology Conference (ESTC), 2016

[14] AdvanSource $\quad$ Biomaterials, "Chronoflex $\mathrm{AR}^{\mathbb{}}$ ", http://www.advbiomaterials.com/products/polycarbonate/ChronoFlex $\% 2$ 0AR.\%20AR-LT.pdf, viewed on 07.06.2018

[15] ASM International, "Materials and Coatings for Medical Devices: Cardiovascular", Materials and processes for medical devices, 2009

[16] D. Ernst, T. Zerna, K. J. Wolter, "Influences of organic materials on packaging technologies and their consideration for lifetime evaluation", International Spring Seminar on Electronics Technology (ISSE), 2011

[17] E.Halonen et al., "Dynamic bending test analysis of inkjet-printed conductors on flexible substrates", 62nd Electronic Components and Technology Conference, 2012

[18] S. Friedrich, "Anwendbarkeit polymererEinhausungsmaterialien für mikroelektronische Implantate", TUDpress, Dresden, 2018

[19] D. Nikolova, "Charakterisierung und Modifizierung der Grenzflächen im Polymer-Metall-Verbund”, Martin-Luther-Universität Halle-Wittenberg, Dissertation, 2005

[20] L.Rebohle, S. Prucnal, W. Skorupa, ,A review of thermal processing in the subsecond range: semiconductors and beyond", Semiconductor Science and Technology, vol. 31 (10), 2016 